\title{
Blood Transfusion Practice in Operating Rooms in Nemazee Hospital in Southern Iran
}

\author{
Sezaneh Haghpanah, MD'; Shima Miladi, MA²; Leila Kasraian, MD³ Ali Zamani, BSc ; Maryam Gholami, PhD ${ }^{2,5^{*}}$ \\ ${ }^{1}$ Hematology Research Center, Shiraz University of Medical Sciences, Shiraz, Iran \\ ${ }^{2}$ Clinical Research Development Center, Nemazee Hospital, Shiraz University of Medical Sciences, Shiraz, Iran \\ ${ }^{3}$ Blood Transfusion Research Center, High Institute for Research and Education in Transfusion Medicine and Shiraz Regional Educational Blood \\ Transfusion Center, Shiraz, Iran \\ ${ }^{4}$ Blood Bank, Nemazee Hospital, Shiraz University of Medical Sciences, Shiraz, Iran \\ ${ }^{5}$ Department of Health Services Management, Science and Research Branch, Islamic Azad University, Tehran, Iran
}

\begin{abstract}
Background: The requests for blood products in elective surgeries exceed actual use, leading to financial wastage and loss of shelf-life. In this study, we assessed the blood transfusion indices in elective surgeries performed in the operating rooms.

Methods: In this cross-sectional study, from January to June 2017, a total of 970 adult patients who underwent elective surgeries in the operating rooms of Nemazee hospital, a general referral hospital in southern Iran, were investigated. Demographic, clinical, and laboratory data, such as hemoglobin $(\mathrm{Hb})$, hematocrit $(\mathrm{Hct})$, platelets, prothrombin time (PT), and partial thromboplastin time (PTT) were gathered from medical records. Blood utilization was evaluated using the following indices: cross-match to transfusion ratio (C/T ratio), transfusion probability (T\%), transfusion index ( $\mathrm{TI}$ ), and Maximum Surgical Blood Order Schedule (MSBOS).

Results: The overall C/T, T\%, and TI ratios were $2.49,46.6 \%$, and 0.83 for all procedures, and the highest and lowest ratios pertained to the thoracic and cardiac surgeries, respectively. The $\mathrm{C} / \mathrm{T}$ ratio was $\geq 2.5$ for all surgical procedures except for cardiac surgeries. $\mathrm{T} \%$ was $<30$ for thoracic and orthopedics surgeries and $\geq 30$ for other surgical procedures. In all surgical procedures, TI was less than 0.5 , except for cardiac surgeries. Also, the MSBOS was about 3 units for cardiac surgeries and ranged from 0.5 to 1 units in other surgeries. Conclusion: The results of this study showed a high quality blood transfusion practice in cardiac surgeries, possibly due to more focus on this critical ward. Assessing difficulties in the process of reservation, utilization, and preparation of standard protocols and policies are required to improve the blood utilization practice in operating rooms.

Keywords: Blood transfusion, Crossmatching, Maximum Surgical Blood Order Schedule (MSBOS)

Cite this article as: Haghpanah S, Miladi S, Kasraian L, Zamani A, Gholami M. Blood transfusion practice in operating rooms in Nemazee hospital in southern Iran. Arch Iran Med. 2021;24(2):107-112. doi: 10.34172/aim.2021.16.
\end{abstract}

Received: December 2, 2019, Accepted: July 22, 2020, ePublished: February 1, 2021

\section{Introduction}

Many surgical patients need blood transfusions due to blood loss during surgery. There are many variations in the transfusion practice. These differences might be caused by differences in patients' characteristics, the amount of perioperative blood loss, and variations in transfusion threshold decisions. ${ }^{1}$

Over-ordering of blood components reduces the stock of blood banks and the shelf-life of blood. Moreover, it may lead to shortage of blood products where blood transfusion requirement is necessary, ${ }^{2}$ resulting in excess financial burden on healthcare expenditure. ${ }^{3-5}$ Several studies have shown that blood units requested for elective surgeries are mostly more than the actual needs. ${ }^{6-8}$ Zaman et al reported that the main cause of inappropriate blood requests in operation rooms is overestimation of blood units by surgical teams. ${ }^{9}$

Several guidelines and strategies have been recommended to modify blood product utilization to develop a cost-effective transfusion practice. ${ }^{10-12}$ To evaluate the transfusion practice, there are some indices including crossmatch to transfusion ratio $(\mathrm{C} / \mathrm{T})$ which was suggested for the first time by Boral Henry in 1975, ${ }^{13}$ transfusion index (TI), and transfusion probability (T\%) introduced by Mead et al in 1980. ${ }^{14}$ Also, Maximum Surgical Blood Order Schedule (MSBOS) was presented to avoid pointless ordering of blood components and reduction of excessive cross-matching and wastage of blood in elective surgeries. MSBOS estimates the maximum number of units of blood that may be needed for each procedure. ${ }^{13-15}$

In this study, we evaluated the blood transfusion practice in elective surgeries in operating rooms of Nemazee hospital, in Shiraz, southern Iran.

\section{Materials and Methods}

A retrospective cross-sectional study, from January to June 2017, was carried out in the operating rooms of Nemazee hospital, a general referral hospital, in Shiraz, southern 
Iran. All adult patients who underwent elective surgical procedures during this six-month period were included in the study $(n=970)$. Pediatric patients were excluded from the study.

Demographic, clinical, and laboratory data, such as age, sex, type of surgery, hemoglobin (Hb), hematocrit (Hct), platelet counts, blood group, prothrombin time (PT), and partial thromboplastin time (PTT) were gathered from medical records. The number of blood units that were requested, cross-matched and transfused, and the number of patients for whom blood was cross-matched or transfused were collected from the patients' records.

\section{Data Analysis}

Data were coded, entered, and analyzed using the Statistical Package for the Social Sciences (SPSS) software version 16. Descriptive data were presented as mean, standard deviation, frequency, and percentage. The Pearson correlation coefficient was calculated to determine the correlation between two quantitative variables. A two-sided $P$ value less than 0.05 was considered to be statistically significant. Blood utilization indices including CT, TI, T\%, and MSBOS indices were calculated based on the formula in Table 1.

\section{Results}

The mean age of the patients was $55.4 \pm 18.2$ years, with $64.1 \%$ being males and $35.9 \%$ females. The demographic characteristics and blood group profiles are shown in Table 2. Blood groups $\mathrm{O}+$ and $\mathrm{AB}$ - had the greatest and smallest frequency with $37 \%$ and $0.7 \%$, respectively. The descriptive information of requested, transfused, and returned blood units (the gap between requested and transfused values) by blood groups is demonstrated in Table 3. The highest and lowest mean number of the requested units were 3.281.25 \pm and $1.870 .81 \pm$ with $95 \%$ CIs [2.13 to 4.44 ] and [1.52 to 2.22], pertaining to ABand A- blood groups, respectively. The highest and lowest mean number of the returned units pertained to the ABand A-blood groups, as well.

Blood request and blood utilization indices including $\mathrm{C} / \mathrm{T}$ ratio, $\mathrm{T} \%$, and $\mathrm{TI}$ for each type of elective surgeries are presented in Table 4 . The overall C/T ratio was 2.49 for all procedures. The highest and lowest $\mathrm{C} / \mathrm{T}$ ratio pertained to thoracic (7.86) and cardiac surgeries (1.54). The total T\% was $46.6 \%$ with the lowest T\% of $16.8 \%$ for thoracic
Table 2. Demographic and Laboratory Data of Surgical Patients

\begin{tabular}{|c|c|}
\hline Variables & Mean $\pm \mathrm{SD}(95 \% \mathrm{Cl})$ \\
\hline Age & $55.40 \pm 18.23(54.23,56.57)$ \\
\hline Hemoglobin (g/dL) & $11.70 \pm 2.46(54.23,56.57)$ \\
\hline Hct $(\%)$ & $36.52 \pm 14.06(35.68,37.51)$ \\
\hline Platelet $/ \mu \mathrm{L}$ & $235000 \pm 104000(228.09,241.51)$ \\
\hline PT (s) & $14.97 \pm 5.60(14.62,15.35)$ \\
\hline \multirow[t]{2}{*}{ PTT (s) } & $33.79 \pm 8.15(33.25,34.31)$ \\
\hline & No. $(\%)$ \\
\hline \multicolumn{2}{|l|}{ Gender } \\
\hline Male & $628(64.1)$ \\
\hline Female & $352(35.9)$ \\
\hline \multicolumn{2}{|l|}{ Blood type and RH } \\
\hline$A+$ & $263(91.6)$ \\
\hline A - & $23(8.0)$ \\
\hline$B+$ & $213(94.7)$ \\
\hline$B-$ & $12(5.3)$ \\
\hline $\mathrm{AB}+$ & $49(87.5)$ \\
\hline$A B-$ & $7(12.5)$ \\
\hline $\mathrm{O}+$ & $350(92.4)$ \\
\hline O - & $28(7.4)$ \\
\hline
\end{tabular}

PT, prothrombin time; PTT, partial thromboplastin time; Hct, hematocrit.

and the highest $\mathrm{T} \%$ of $80.1 \%$ for cardiac surgeries. The total TI was 0.83 . Thoracic and cardiac surgeries were the procedures with the lowest and the highest TI $(0.25$ and 1.75), respectively.

The C/T ratio was $\geq 2.5$ for all surgical procedures except for cardiac surgeries. T\% was $<30$ for thoracic and orthopedics surgeries and $\geq 30$ for other surgical procedures. TI was less than 0.5 in all surgical services except for cardiac surgeries (1.75).

The highest MSBOS was 2.62 units for cardiac surgeries and the lowest was 0.37 units for thoracic surgeries. This variable ranged from 0.55 to 0.69 units for other surgeries.

Table 5 presents the correlation of the requested, transfused and returned blood units with laboratory data of surgical patients. Significant relationships were observed between platelets $(\mathrm{r}=-0.185, P<0.001)$ and PT $(\mathrm{r}=0.092$, $P=0.004)$ with the requested blood. On the other hand, significant relationships were observed between $\mathrm{Hb}(\mathrm{r}=$ -0.114, $P<0.001)$, platelets $(\mathrm{r}=-0.170, P=0.001)$ and PT ( $\mathrm{r}=0.082, P=0.011)$ with transfused blood. Also, we found a significantly positive correlation of $\mathrm{Hb}(\mathrm{r}=0.177$, $P<0.001)$, and Hct $(\mathrm{r}=0.076, P=0.018)$ with returned

Table 1. Formula and Standard Values of Blood Utilization Indices

\begin{tabular}{|c|c|c|}
\hline Indices & Formula & Appropriate Value \\
\hline $\mathrm{C} / \mathrm{T}$ ratio & Number of units cross-matched/Number of units transfused & $<2.5$ \\
\hline $\mathrm{T} \%$ & Number of patients transfused $* 100 /$ Number of patients cross-matched & $\geq 30 \%$ \\
\hline $\mathrm{TI}$ & Number of units transfused/Number of patients cross-matched & $>0.5$ \\
\hline MSBOS & $\mathrm{TI} * 1.5$ & Institution-specific \\
\hline
\end{tabular}

$\mathrm{C} / \mathrm{T}$ ratio, cross-match to transfusion ratio; T\%, transfusion probability; TI, transfusion Index, MSBOS, Maximum Surgical Blood Order Schedule. 
Table 3. Requested, Transfused and Returned Blood Based on Blood Groups of Surgical Patients

\begin{tabular}{|c|c|c|c|}
\hline Blood Type, Rh & $\begin{array}{l}\text { Requested } \\
\text { Blood Units }\end{array}$ & $\begin{array}{l}\text { Transfused } \\
\text { Blood Units }\end{array}$ & $\begin{array}{c}\text { Returned } \\
\text { Blood Units }\end{array}$ \\
\hline & Mean \pm SD $(95 \% \mathrm{Cl})$ & Mean $\pm \mathrm{SD}(95 \% \mathrm{CI})$ & Mean Difference $\pm \mathrm{SD}(95 \% \mathrm{Cl})$ \\
\hline$A+$ & $2.33 \pm 1.12(2.19,2.47)$ & $0.89 \pm 1.09(0.76,1.03)$ & $1.43 \pm 0.93(1.32,1.54)$ \\
\hline A - & $1.87 \pm 0.81(1.52,2.22)$ & $0.48 \pm 0.79(0.14,0.82)$ & $1.39 \pm 0.94(0.98,1.80)$ \\
\hline$B+$ & $2.36 \pm 1.13(2.21,2.52)$ & $0.83 \pm 1.06(0.69,0.98)$ & $1.53 \pm 1.07(1.38,1.68)$ \\
\hline B - & $2.67 \pm 1.43(1.75,3.58)$ & $1.25 \pm 0.87(0.70,1.80)$ & $1.42 \pm 1.38(0.54,2.29)$ \\
\hline $\mathrm{AB}+$ & $2.17 \pm 0.94(1.89,2.43)$ & $0.57 \pm 0.91(0.31,0.83)$ & $1.59 \pm 0.91(1.33,1.85)$ \\
\hline $\mathrm{AB}-$ & $3.28 \pm 1.25(2.13,4.44)$ & $1.14 \pm 1.34(-0.10,2.39)$ & $2.14 \pm 1.21(1.02,3.27)$ \\
\hline $\mathrm{O}+$ & $2.48 \pm 1.33(2.33,2.61)$ & $0.82 \pm 1.15(0.70,0.95)$ & $1.66 \pm 1.22(1.53,1.79)$ \\
\hline O - & $2.11 \pm 1.18(1.64,2.59)$ & $0.61 \pm 0.90(0.25,0.98)$ & $1.50 \pm 1.21(1.01,1.99)$ \\
\hline
\end{tabular}

Table 4. Blood Transfusion Indices in Various Surgical Services of Nemazee Hospital

\begin{tabular}{|c|c|c|c|c|c|c|c|c|}
\hline \multirow{2}{*}{ Surgical Services } & \multicolumn{2}{|c|}{ Cross-matched } & \multicolumn{2}{|c|}{ Transfused } & \multirow{2}{*}{ CT } & \multirow{2}{*}{$\mathbf{T} \%$} & \multirow{2}{*}{$\mathrm{TI}$} & \multirow{2}{*}{ MSBOS } \\
\hline & No. of Patients (\%) & No. of Units (\%) & No. of Patients (\%) & No. of Units (\%) & & & & \\
\hline Cardiac, $\mathrm{n}=307$ & $307(32.1)$ & 825 (41.5) & $246(55.4)$ & $537(67.5)$ & 1.54 & 80.1 & 1.75 & 2.62 \\
\hline Kidney, $\mathrm{n}=207$ & $204(21.3)$ & 376 (18.9) & $67(15.1)$ & 95 (11.9) & 3.96 & 32.8 & 0.46 & 0.69 \\
\hline Orthopedics, $n=51$ & $49(5.1)$ & $91(4.6)$ & $13(2.9)$ & $18(2.3)$ & 5.05 & 26.5 & 0.37 & 0.55 \\
\hline Plastic, $\mathrm{n}=84$ & $84(8.8)$ & $143(7.2)$ & $31(7.0)$ & $39(5.0)$ & 3.67 & 36.9 & 0.46 & 0.69 \\
\hline General, $n=148$ & $145(15.2)$ & $249(12.5)$ & $50(11.3)$ & $56(7.0)$ & 4.45 & 34.5 & 0.38 & 0.57 \\
\hline Neuro surgery, $n=53$ & $51(5.3)$ & $75(3.8)$ & $18(4.0)$ & $23(2.9)$ & 3.26 & 35.3 & 0.45 & 0.67 \\
\hline Thoracic, $n=114$ & $113(11.8)$ & $220(11.1)$ & $19(4.3)$ & $28(3.5)$ & 7.86 & 16.8 & 0.25 & 0.37 \\
\hline
\end{tabular}

T\%, transfusion probability; TI, transfusion Index, MSBOS, Maximum Surgical Blood Order Schedule.

Table 5. Pearson Correlation Coefficients between Requested, Transfused, and Returned Blood Units with Age and Laboratory Data in Surgical Patients

\begin{tabular}{|c|c|c|c|c|c|c|c|}
\hline Variables & & Age (y) & $\mathrm{Hb}(\mathrm{g} / \mathrm{dL})$ & Hct $(\%)$ & $\operatorname{Plt}(/ \mu \mathrm{L})$ & PT (s) & PTT (s) \\
\hline \multirow{2}{*}{ Requested blood units } & Correlation coefficient $r$ & 0.148 & 0.60 & 0.019 & -0.185 & 0.092 & 0.003 \\
\hline & $P$ value & $<0.001^{*}$ & 0.063 & 0.561 & $<0.001^{*}$ & $0.004^{*}$ & 0.926 \\
\hline \multirow{2}{*}{ Transfused blood units } & Correlation coefficient $r$ & 0.210 & -0.114 & -0.057 & -0.170 & 0.082 & 0.026 \\
\hline & $P$ value & $<0.001^{*}$ & $<0.001^{*}$ & 0.079 & $<0.001^{*}$ & $0.011^{*}$ & 0.425 \\
\hline \multirow{2}{*}{ Returned blood units } & Correlation coefficient $r$ & -0.048 & 0.177 & 0.076 & -0.035 & 0.018 & -0.018 \\
\hline & $P$ value & 0.145 & $<0.001^{*}$ & $0.018^{*}$ & 0.273 & 0.574 & 0.571 \\
\hline
\end{tabular}

* Statistically significant.

Hb, Hemoglobin (g/dL); Hct, hematocrit; Plt, platelet; PT, prothrombin time (s); PTT, partial thromboplastic time (s).

blood. Moreover, the patients' age showed a mild positive correlation with both requested $(\mathrm{r}=0.148)$ and transfused blood $(r=0.182)(P<0.001)$.

\section{Discussion}

Blood and its components play an important role in maintaining the survival of patients undergoing elective surgeries. In addition to the shortage of this vital substance, nowadays we face other problems around the world such as rising demands and requested blood products remaining unused. Excessive blood requests, besides imposing a high financial burden on the health system, can reduce the blood supply and impair the process of blood transfusion to patients. Taken together, it negatively affects the patients undergoing surgical procedures. ${ }^{16,17}$

Blood transfusion indices such as CT, T\% and TI can also be affected by many factors including differences in surgical and anesthetic techniques, preoperative conditions of the patients and variations in the usage of blood due to different transfusion protocols. ${ }^{12,13}$

Based on our results, the maximum cross-matched blood units pertained to cardiac surgery service followed by kidney and general surgery units. Cardiac surgery contributed to $41.5 \%$ of the total blood units crossmatched among all surgical procedures, of which $34.9 \%$ were unused. Overall, the present study revealed that $59.9 \%$ of the cross-matched blood was unused. This finding is nearly similar to that reported in Ethiopian 
(56.4\%), and Indian (60.3\%) studies. ${ }^{6,18}$ However, it is less than the values reported in other poor countries such as Sri Lanka (84.9\%), Pakistan (95.1\%), and Tanzania $(82.9 \%) .{ }^{19-21}$ In three Iranian studies, greater wastage of blood components was reported, ${ }^{22-24}$ while it was less in one study conducted in Iran. ${ }^{25}$ In our study, the amount of requested and transfused blood was positively correlated with age and preoperative value of PT and negatively correlated with preoperative values of $\mathrm{Hb}$ and platelet of the surgical patients. Besides, the amount of returned blood showed a positive correlation with $\mathrm{Hb}$ and HCT, indicating that higher preoperative $\mathrm{Hb}$ level is related to less blood transfusion need and a higher amount of blood wastage. Our results were in contrast with those of Yazdi et al who observed no relationship between these preoperative factors and transfusion needs. ${ }^{24}$

A CT ratio of 2.5 or below, T\% of $\geq 30 \%$, and TI of more than 0.5 are indicative of efficient blood utilization. The overall CT ratio of 2.49 in the present study was relatively higher than those previously reported in other studies in the same settings ${ }^{25-29}$ and it was very similar to the ratio reported in India. ${ }^{18}$ However, it was lower than what was reported in Egypt (3.9), Malaysia (5), Tanzania (5.8), Thailand (4.27), 2,19,30,31 and also some studies in Iran (ranging from 3.71 to 7.38 ) which could be attributed to their inappropriate use of MSBOS guidelines. ${ }^{22-24}$ The $\mathrm{C} / \mathrm{T}$ ratio of more than 2.5 in all surgical services in our study, except for cardiac surgery service, highlights our inappropriate blood utilization. The highest CT was observed in thoracic surgery (7.86), compared to studies conducted in Iran, India and the United Kingdom which reported lower CT ratios (1.53, 2.58 and 1.2-3, respectively). ${ }^{24,28,32}$ The reason for the high CT ratio can be the tendency to request blood units more than the real requirement. Over-ordering of blood by physicians may be a result of the extreme request of blood by surgeons during surgery to ensure that in case of an unexpected blood loss, the patient would be safe.

The results of this study revealed an overall transfusion probability (T\%) of $46.6 \%$ which was indicative of over-reservation of blood units. This finding was almost similar to that reported in the Ethiopian study ${ }^{6}$ and was more than what was observed in researches carried out in Tanzania (15.9\%), Thailand (23\%), Egypt (36.9\%), 2,19,3 and previous Iranian studies $(16.83 \%, 14.52 \%$, and $12.8 \%$, respectively). ${ }^{22-24}$ This is indicative of appropriate utilization of blood units in our hospital compared to those studies, whereas several Indian studies showed higher indices. ${ }^{26-29}$ Also among the mentioned surgeries, this ratio was $16.8 \%$ for thoracic surgeries which is lower than what was reported in some countries such as the UK (50\%), Iran $(52.95 \%)$, and India $(63.64 \%)$ as well as $80.1 \%$ in cardiac surgeries which is in line with studies by Kaur et al (89.75\%) and Mangwana et al (85.84\%) in India. ${ }^{24,26,28,32}$

The TI ratio in the current study was 0.83 , indicating efficient blood usage. This index is higher than TI ratios determined in other studies conducted in Thailand (0.23), Tanzania (0.2), Sri Lanka $(<0.5)$, and India $(0.5) .^{2,19,20,27}$ Similarly, it was higher than the TI values reported in some previous Iranian studies. ${ }^{22-24}$ However, several researchers have found an overall TI ratio of more than one..$^{25,26,28}$ This illustrates better utilization management compared to ours. On the other hand, there were other studies from Ethiopia ${ }^{6}$ and India, ${ }^{29}$ reporting TI ratios of 0.77 and 0.81 , respectively, which are comparable to our finding. Based on our results, cardiac surgeries had the highest TI value (1.75) which was higher than the value determined in some analogous studies which were previously mentioned (0.5). ${ }^{22,27}$ Likewise, the highest TI value in one of the Iranian studies pertained to cardiac surgeries (4.03). ${ }^{25}$ In our study, the lowest TI value pertained to thoracic surgeries (0.25). It was even less than what was reported in operating rooms of multiple countries including Iran $\left(0.88,1.1,1.2\right.$, respectively). ${ }^{24,28,32}$ The calculated ratio of TI for all other surgical procedures is less than 0.5 , which indicates blood compatibility testing is not required before the surgery.

Through the use of Mead's criterion, the MSBOS is 1.5 times the TI for each surgical procedure. In our study, the highest and lowest MSBOS pertained to cardiac and thoracic surgeries $(2.62,0.37)$, respectively, which is comparable to the studies mentioned above. (It was 0.75 in Dashab et al and Kuchhal et al; 6.04 in Nikpour et al; and 1.32, 1.65 and 1.8 in Yazdi et al, Mangwana et al, and Charles et al, respectively). ${ }^{22,24,25,27,28,32}$

The blood conservation indices should be used to monitor unessential orders and overall requisition of blood. The reduction of unnecessary perioperative blood orders by MSBOS contributes to the modification of extra cost in hospitals and decreases the financial charge of the patients. One study in the United Kingdom showed that the use of MSBOS protocol resulted in a considerable improvement in blood ordering with a reduction in CT ratios. ${ }^{33}$ Also, similar studies in the United States in 2013 and 2014 showed cost-savings of $\$ 254.583$ and $\$ 137.223$, respectively. ${ }^{10,34}$ In addition, another study in the United Kingdom demonstrated an annual saving of about $£ 8596.00$. $^{7}$ Furthermore, in Nigeria, India, and Thailand, a reduction in hospital costs was reported through implementation of MSBOS. ${ }^{35-37}$ This highlights an urgent need to formulate an appropriate blood ordering schedule by blood banks in the hospital.

In conclusion, although overall CT, TI, and T\% indices were compatible with standard values, CT ratio and TI values were not compatible with the standard range in each of the surgical services, except for cardiac surgery service. $\mathrm{T} \%$ indices were in the standard range in all services, except for thoracic and orthopedic services. This shows a high quality blood transfusion practice in cardiac surgery, possibly due to more focus on this critical ward. Situation 
analysis and qualitative research are required to find the basic problems in transfusion practice in different surgical services and preparation of a standard protocol to improve the blood utilization practice. It seems that the estimation of MSBOS according to our setting can improve blood ordering.

\section{Authors' Contribution}

$\mathrm{SH}$ and $\mathrm{MGH}$ developed the concept for the manuscript, drafted and revised the manuscript. SHM conducted data analysis and data gathering, and drafted the manuscript. LK drafted and revised the manuscript. AZ cooperated in data gathering and drafted the manuscript. All authors read and approved the final manuscript.

\section{Conflict of Interest Disclosures}

The authors reported that they have no conflict of interest.

\section{Ethical Statement}

The study protocol was approved by the Ethical Committee of Shiraz University of Medical Sciences (Approval code $=15398.138907$ ).

\section{Acknowledgements}

The authors would like to thank Shiraz University of Medical Sciences, Center for Development of Clinical Research of Nemazee Hospital and Dr. Nasrin Shokrpour for editorial assistance. Shiraz Blood Transfusion Organization is also acknowledged for approval and financial supports (Grant No. 15398-138907). We also appreciate Marzieh Khojastehfar, Gholamreza Farhadi and Narges Rezaei who helped us in gathering the data.

\section{References}

1. Gombotz H, Rehak PH, Shander A, Hofmann A. Blood use in elective surgery: the Austrian benchmark study. Transfusion. 2007;47(8):1468-80. doi: 10.1111/j.15372995.2007.01286.x.

2. Boriboonhirunsarn D, Chaopothong $\mathrm{P}$, Jirasawas $\mathrm{T}$. Blood Transfusion in Elective Abdominal Gynecologic Surgery. J Gynecol Surg. 2017;33(6):231-5. doi: 10.1089/ gyn.2017.0004.

3. Berwick DM, Hackbarth AD. Eliminating waste in US health care. JAMA. 2012;307(14):1513-6. doi: 10.1001/ jama.2012.362.

4. Collins RA, Wisniewski MK, Waters JH, Triulzi DJ, Yazer MH. Effectiveness of multiple initiatives to reduce blood component wastage. Am J Clin Pathol. 2015;143(3):329-35. doi:10.1309/ AJCP42WMHSSTPHXI.

5. Gharah BA, Jalilzadeh KM, Honarkaran N, Davoodi F. Estimsation and comparison of the production cost of blood and blood products in 28 IBTO centers in 2002. Sci J Iran Blood Transfus Organ. 2005;1(2):61-70.

6. Belayneh T, Messele G, Abdissa Z, Tegene B. Blood requisition and utilization practice in surgical patients at university of Gondar hospital, northwest Ethiopia. J Blood Transfus. 2013:2013:758910. doi: 10.1155/2013/758910.

7. Hall TC, Pattenden C, Hollobone C, Pollard C, Dennison AR. Blood transfusion policies in elective general surgery: how to optimise cross-match-to-transfusion ratios. Transfus Med Hemother. 2013;40(1):27-31. doi: 10.1159/000345660.

8. Singh JK, Singh P. Routine pre-operative cross-match for elective colorectal resections: An appropriate use of resources? Surgeon. 2011;9(1):8-12. doi: 10.1016/j.surge.2010.06.008.

9. Zaman B, Radmehr M, Sahraian A, Sohrabi P. Determination of the ratio and causes of unused blood ordered from blood bank blood in elective surgery in Rasoul-e-Akram hospital. Sci J Iran Blood Transfus Organ. 2009;6(2):141-6.

10. Frank SM, Oleyar MJ, Ness PM, Tobian AA. Reducing unnecessary preoperative blood orders and costs by implementing an updated institution-specific maximum surgical blood order schedule and a remote electronic blood release system. Anesthesiology. 2014;121(3):501-9. doi: 10.1097/ALN.0000000000000338.

11. Murphy W, Phillips P, Gray A, Heatley L, Palmer J, Hopkins D, et al. Blood use for surgical patients: a study of Scottish hospital transfusion practices. J R Coll Surg Edinb. 1995;40(1):10-3.

12. Richardson NG, Bradley WN, Donaldson DR, O'Shaughnessy DF. Maximum surgical blood ordering schedule in a district general hospital saves money and resources. Ann R Coll Surg Engl. 1998;80(4):262-5.

13. Friedman B, Oberman H, Chadwick A, Kingdon K. The maximum surgical blood order schedule and surgical blood use in the United States. Transfusion. 1976;16(4):380-7. doi: 10.1046/j.1537-2995.1976.16476247063.x.

14. Mead JH, Anthony CD, Sattler M. Hemotherapy in elective surgery: an incidence report, review of the literature, and alternatives for guideline appraisal. Am J Clin Pathol. 1980;74(2):223-7. doi: 10.1093/ajcp/74.2.223.

15. Dodsworth H, Dudley H. Increased efficiency of transfusion practice in routine surgery using pre-operative antibody screening and selective ordering with an abbreviated crossmatch. Br J Surg. 1985;72(2):102-4. doi: 10.1002/ bjs. 1800720210 .

16. Shander A, Hofmann A, Ozawa S, Theusinger OM, Gombotz $\mathrm{H}$, Spahn DR. Activity-based costs of blood transfusions in surgical patients at four hospitals. Transfusion. 2010;50(4):75365. doi: 10.1111/j.1537-2995.2009.02518.x.

17. Vibhute M, Kamath SK, Shetty A. Blood utilisation in elective general surgery cases: requirements, ordering and transfusion practices. J Postgrad Med. 2000;46(1):13-7.

18. Subramanian A, Sagar S, Kumar S, Agrawal D, Albert V, Misra MC. Maximum surgical blood ordering schedule in a tertiary trauma center in northern India: A proposal. J Emerg Trauma Shock. 2012;5(4):321-7. doi: 10.4103/0974-2700.102391.

19. Chalya PL, Mbunda F, Mabula JB, Massinde AN, Kihunrwa A, Gilyoma JM. Blood transfusion practice in surgery at Bugando Medical Centre in northwestern Tanzania. Tanzan J Health Res. 2016;18(1). doi: 10.4314/thrb.v18i4.

20. Gamage C, Pratheepan P, Sivaganesh S. Rationale for blood request: cross match versus group and screen. Sri Lanka Journal of Surgery. 2014;31(3). doi: 10.4038/sljs.v31i3.6225.

21. Soomro R, Javed M, Ali S. Arrangements and use of blood in elective surgical procedures. Professional Med J. 2011;18(2):212-4.

22. Dashab M, Solhjou K, Erfanian S. Comparing the demand for blood in hospitals of Jahrom and standard blood transfusion indices. J Jahrom Univ Med Sci. 2014;12(1):63-9. doi: 10.29252/jmj.12.1.63.

23. Sheikhansari S, Darbandi B, Zahiri Sorouri Z, Bagheralimi A. Evaluating Blood Requests and Transfusion Practice in Major Surgical Procedures. Iran J Blood Cancer. 2015;7(5):227-30.

24. Yazdi AP, Alipour M, Jahanbakhsh SS, Gharavifard M, Gilani MT. A survey of blood request versus blood utilization at a university hospital in Iran. Arch Bone Jt Surg. 2016;4(1):75-9.

25. Nikpoor A, Daneshvar H, Sanei Moghaddam E, Askari M. Assessment of requisition and consumption indices of blood in educational hospitals in Kerman city. Sci J Iran Blood Transfus Organ. 2013;10(1):12-19.

26. Kaur D, Kandwal M. Blood utilization and quality indicatorstrend at a super speciality hospital in Northern India. Hematol Transfus Int J. 2016;2(3):00037. doi: 10.15406/ htij.2016.02.00037.

27. Kuchhal A, Negi G, Gaur D, Harsh M. Blood utilization practices in elective surgical patients in a Tertiary Care Hospital of Uttarakhand. Glob J Transfus Med. 2016;1(2):516. doi: 10.4103/2455-8893.189843.

28. Mangwana S, Bedi N, Yadav P, Chugh R. Optimization of blood transfusion services: Analysis of blood requisition and 
utilization practices in cardiac surgical patients in a tertiary care hospital, India. Glob J Transfus Med. 2017;2(1):47-51. doi: 10.4103/2468-8398.202713.

29. Yasmeen I, Ahmed I, Sidhu M. Pattern of blood component cross-matching and their utilization in a tertiary care hospital of Jammu region. Int J Res Med Sci. 2018;6(4):1337-41. doi: 10.18203/2320-6012.ijrms20181293.

30. Ibrahim SZ, Mamdouh HM, Ramadan AM. Blood utilization for elective surgeries at main University Hospital in Alexandria, Egypt. J Am Sci. 2011;7(6):683-9.

31. Jayaranee S, Prathiba R, Vasanthi N, Lopez CG. An analysis of blood utilization for elective surgery in a tertiary medical centre in Malaysia. Malays J Pathol. 2002;24(1):59-66.

32. Charles K, De Freitas L, Ramoutar R, Goolam R, Juman S, Murray $\mathrm{D}$, et al. Blood utilization in a developing society: what is the best index of efficiency? Transfus Med. 2018;28(6):41319. doi: 10.1111/tme.12534.

33. Mahadevan D, Challand C, Clarke A, Keenan J. Maximum surgical blood ordering schedules for revision lower limb arthroplasty. Arch Orthop Trauma Surg. 2011;131(5):663-7. doi:10.1007/s00402-010-1204-2.

34. Frank SM, Rothschild JA, Masear CG, Rivers RJ, Merritt WT, Savage WJ, et al. Optimizing preoperative blood ordering with data acquired from an anesthesia information management system. Anesthesiology. 2013;118(6):1286-97. doi: 10.1097/ ALN.0b013e3182923da0.

35. Hardy NM, Bolen FH, Shatney $\mathrm{CH}$. Maximum surgical blood order schedule reduces hospital costs. Am Surg. 1987;53(4):223-5.

36. Mahar FK, Moiz B, Khurshid M, Chawla T. Implementation of Maximum Surgical Blood Ordering Schedule and an Improvement in Transfusion Practices of Surgeons subsequent to Intervention. Indian J Hematol Blood Transfus. 2013;29(3):129-33. doi: 10.1007/s12288-012-0169-4.

37. Saringcarinkul A, Chuasuwan S. Maximum Surgical Blood Order Schedule for Elective Neurosurgery in a University Teaching Hospital in Northern Thailand. Asian J Neurosurg. 2018;13(2):329-35. doi: 10.4103/ajns.AJNS_104_16. 